\title{
ESTRÉS LABORAL EN PERSONAL MÉDICO Y ENFERMERÍA DE ATENCIÓN PRIMARIA ANTE LA EMERGENCIA SANITARIA POR COVID-19.
}

\section{Job stress in primary care medical and nursing personnel in the COVID-19 health emergency.}

\author{
${ }^{1}$ Betzy Evelyn Moncada Rodríguez iD , ${ }^{1}$ Mónica Paola Suárez Lluccha (iD, , ${ }^{1}$ Luis Duque Cordova \\ (iD), ${ }^{2}$ Kenny Fernando Escobar Segovia* (iD \\ ${ }^{1}$ Universidad de Especialidades Espíritu Santo, Facultad de Postgrado, Guayaquil, Ecuador. \\ ${ }^{2}$ Escuela Superior Politécnica del Litoral, Guayaquil, Ecuador. \\ *kescobar@espol.edu.ec
}

$\mathrm{R}$ esumen

El estrés laboral implica un conjunto de reacciones fisiológicas, cognitivas, emocionales y conductuales, que el individuo experimenta ante circunstancias que implican excesiva presión en un contexto laboral. Esta situación puede repercutir negativamente en el desempeño, la motivación, la satisfacción y el compromiso del trabajador con la empresa. Considerando la complejidad de las condiciones laborales impuestas por COVID-19 al personal sanitario, el objetivo del presente estudio fue analizar el estrés laboral entre profesionales sanitarios de atención primaria (médicos/as y enfermeros/as) que trabajan en un Distrito de Salud del Ministerio de Salud Pública del Ecuador durante la emergencia sanitaria por COVID-19. El estudio es de tipo cuantitativo de corte transversal, se aplicó a una muestra no probabilistica a conveniencia de 41 personas un cuestionario de variables sociodemográficas y el cuestionario de estrés laboral (JSS). Ambos tipos de profesionales presentan niveles elevados de estrés laboral, siendo los médicos quienes obtienen las puntuaciones más altas y personas mayores a 30 años. Es indispensable profundizar en este tipo de análisis para obtener datos que permitan toma de decisiones acertadas que eviten pérdida de vidas, desgaste innecesario del personal sanitario y la disminución de su productividad.

Palabras claves: Personal sanitario, COVID-19, Salud mental.

\section{A bstract}

Job stress involves a set of physiological, cognitive, emotional and behavioral reactions that the individual experiences when faced with circumstances that imply excessive pressure in a work context. This situation can have a negative impact on the performance, motivation, satisfaction and commitment of the worker with the company. Considering the complexity of the working conditions imposed by COVID-19 on health personnel, the objective of this study was to analyze work stress among primary care health professionals (doctors and nurses) who work in a Health District of the Ministry of Public Health of Ecuador during the health emergency due to COVID-19. The study is of a quantitative cross-sectional type; a questionnaire of sociodemographic variables and the Job Stress Questionnaire (JSS) were applied to a non-probabilistic convenience sample of 41 people. Both types of professionals present high levels of work stress, with doctors obtaining the highest scores and people over 30 years of age. It is essential to deepen this type of analysis in order to obtain data that will allow for sound decision making to avoid loss of life, unnecessary wear and tear on healthcare personnel and a decrease in their productivity.

Keywords: Health personnel, COVID-19, Mental health. 


\section{INTRODUCCIÓN}

El estrés laboral puede definirse como un conjunto de reacciones fisiológicas, cognitivas, emocionales y conductuales que el individuo experimenta ante circunstancias que implican excesiva presión o demanda de respuesta en un determinado contexto laboral (1). Se trata de un estado que puede producir alteraciones físicas y mentales que repercuten negativamente en el desempeño, la motivación, la satisfacción y el compromiso del trabajador, $y$, por consiguiente, en el clima laboral de la empresa (2-3). Estas consecuencias han dado lugar al desarrollo de numerosos estudios que pretenden explicar la relación entre las demandas de las organizaciones o empresas y los recursos del individuo para afrontarlas.

Este acercamiento al análisis del estrés laboral y su desarrollo se puede explicar mejor mediante tres modelos. El primero, es el modelo ajuste persona-ambiente, que establece que debe existir una relación entre la persona y el ambiente laboral en el que se desenvuelve, es decir, se trata de encontrar un balance entre las necesidades del trabajador y los suministros disponibles para poder cumplir con tales necesidades (2). El segundo, es el modelo demanda-control, que plantea dos dimensiones (demanda y control), a partir de las cuales se considera que las demandas no son estresantes si se combinan con un control adecuado sobre el trabajo y el ambiente. Por lo tanto, la tensión, la fatiga, la ansiedad, la depresión y las enfermedades físicas se deben al desajuste entre el desempeño del trabajo que se realiza y la capacidad que tiene el trabajador para controlar esa demanda (3). Finalmente, el tercero es el modelo esfuerzo-recompensa, que determina las condiciones de riesgo laboral cuando hay presencia de carga de trabajo, demandas y obligaciones del puesto de trabajo, frente a las estrategias que la organización establece para que el trabajador pueda afrontar las demandas y recibir beneficios (4). A partir de estos modelos han surgido diferentes instrumentos destinados a analizar el estrés ocupacional desde diferentes niveles y dimensiones, algunos de los cuales se explican a continuación, por su relevancia en este campo de estudio: 1) Cuestionario sobre el contenido del trabajo [Job Content Questionnaire] (5), 2) Cues- tionario de Estrés Laboral Genérico del NIOSH [Generic Job Stress Questionaire] (6), y 3) Cuestionario de Estrés Laboral (JSS) (7).

El cuestionario de Estrés Laboral JSS (7), se trata de la adaptación española del Job Stress Survey (8), diseñada para medir el nivel general de estrés laboral en adultos. Este cuestionario cuenta con tres índices (estrés laboral, presión laboral y falta de apoyo de la organización), a partir de los cuales se analiza la severidad y la frecuencia con que se presentan 30 eventos estresores. Esta información se obtiene mediante una lista de 60 situaciones relacionados con el trabajo, que tiene una duración de 15-20 minutos y debe ser aplicada de manera individualizada.

En el contexto de este estudio, es importante mencionar algunos de los elementos que pueden incidir en el estrés laboral y, a su vez, en la presencia de alteraciones físicas y mentales, el ambiente de trabajo, la organización y administración de una actividad, los sistemas de trabajo y la calidad de las relaciones humanas (9-10). Estos factores pueden deberse a la presencia de trabajos con gran demanda de atención y responsabilidad, amenaza de demandas laborales, liderazgo inadecuado, rotación de turnos, jornada de trabajo excesiva y actividades físicas corporales inadecuadas, que influyen negativamente en el rendimiento laboral y el bienestar del individuo. En lo posible, se debe evitar que el profesional llegue a la insatisfacción profesional y familiar, que habitualmente se ve reflejada en actitudes de agresividad, inadecuadas relaciones interpersonales en el ámbito laboral y familiar, etc. (11). La calidad de la respuesta de la persona a factores que generan estrés puede proteger su salud integral o generar, agravar y cronificar patologías físicas y mentales. Una de las consecuencias del estrés laboral es el síndrome de Burnout, que corresponde a un estado de agotamiento físico y psicológico, que se desarrolla de manera progresiva, cuya principal consecuencia en el contexto laboral es el desinterés del trabajador por su función, sus tareas y sus compromisos, dando lugar a un peor rendimiento general y, como consecuencia, a pobres indicadores de la organización en cuanto a productividad y cumplimiento de sus objetivos (12-14). 
Actualmente, la pandemia por COVID-19 parece haber agravado los niveles de estrés laboral presente en trabajadores de diversas áreas, incluida la sanitaria. En este ámbito, y dadas las características de las medidas tomadas por los gobiernos, en orden a prevenir el contagio y atender debidamente al usuario en las unidades de salud, el personal sanitario, especialmente médicos/as y enfermeros/as, por su papel en el servicio que brindan al paciente, se ha visto expuesto a un conjunto de situaciones, factores y limitaciones que representan una carga de estrés añadida a la habitual, como son: condiciones inseguras, escases de equipos de protección personal, falta de medicamentos, medidas de seguridad estrictas y necesidad de concentración, entre otros. En este sentido, estudios recientes han resaltado algunos de los principales factores relacionados con el agravamiento del estrés laboral y sus consecuencias en el personal sanitario, en el marco de la pandemia por COVID-19: agotamiento físico mental, complejidad en la toma de decisiones difíciles y en la detección de pacientes sospechosos, sufrimiento por la pérdida de pacientes y compañeros de trabajo, etc. La afectación psicológica se presenta, de modo más frecuente, con sintomatología de ansiedad, fatiga, traumas y depresión (15-18).

Las condiciones laborales a nivel hospitalario causan problemas mentales en el personal sanitario, como estrés, ansiedad, síndrome de depresión, insomnio, ira y miedo. El personal médico que mantiene contacto directo con pacientes COVID-19 manifiestan un alto nivel de ansiedad que es relacionado con el estrés y reducción de la calidad del sueño, angustia, reacciones exageradas que puede recurrir a trastornos depresivos, estrés postraumáticos etc. (19).

Es oportuno señalar que, la forma en que se ha presentado la COVID-19 y el impacto que ha generado en la seguridad y el desarrollo de cada sociedad, guarda relación con los recursos disponibles de una nación y las estrategias que aplica cada gobierno para afrontar esta pandemia y evitar, primordialmente, la pérdida de vidas, el desgaste innecesario del personal sanitario y la disminución evitable de productividad. A pesar de la necesidad de contar con información, que permita a las instancias ministeriales competentes en el contexto laboral sanitario (p.ej., Ministerio de Salud Pública, Ministerio del Trabajo), diseñar estrategias y tomar medidas dirigidas a obtener mejores resultados en esta materia, hasta la fecha no se han desarrollado estudios sobre el estrés laboral del personal sanitario ecuatoriano en los meses de confinamiento por motivo de la COVID-19.

En función de lo referido, por los elevados niveles de estrés en el personal sanitario que está causando la situación actual como es la enfermedad COVID-19, el objetivo del presente estudio fue estudiar las diferencias de estrés laboral entre profesionales sanitarios de atención primaria ante la emergencia sanitaria, que trabajan en un Distrito de Salud del Ministerio de Salud Pública del Ecuador, utilizando como instrumento de estudio el cuestionario de variables sociodemográficas y el cuestionario de estrés laboral (JSS) que mide el nivel general de estrés laboral, como la severidad y la frecuencia con que ocurren 30 fuentes genéricas de estrés (equivalencia de puntuaciones directas en percentiles (Pc), considerándose como hipótesis que estos profesionales presentarán un nivel elevado de estrés laboral, siendo éste de mayor magnitud en los/as médicos/as, porque se asume que desempeñan un rol de mayor responsabilidad en el entorno sanitario.

\section{MATERIALES Y MÉTODOS}

Se trata de un estudio cuantitativo de corte transversal.

\section{Participantes}

La muestra de este estudio fue de tipo no probabilística a conveniencia y estuvo compuesta por 41 profesionales sanitarios de atención primaria que trabajan en unidades de un Distrito de salud del Ministerio de Salud Pública del Ecuador, en la provincia de Guayas.

\section{Criterios de inclusión}

Los criterios de inclusión fueron: (a) ser médico/a o enfermero/a, (b) trabajar actualmente en 
unidades de salud de atención primaria de un Distrito de Salud de la provincia del Guayas, (c) haber prestado atención profesional personalizada en el lapso de marzo a octubre de 2020, datos obtenidos mediante encuestas sociodemográficas realizadas por escrito por cada profesional (d) conocer las características del estudio, y (e) firmar el consentimiento informado.

\section{Instrumentos y medidas}

Para la obtención de datos necesarios para el presente estudio, se diseñó un protocolo de evaluación compuesto por dos instrumentos: 1) Cuestionario ad hoc de variables sociodemográficas y de salud, y 2) Cuestionario de Estrés Laboral (JSS) (7).

Cuestionario ad hoc de variables sociodemográficas y de salud.

Se diseñó un cuestionario, cuya aplicación requiere aproximadamente 15 minutos, que mide variables sociodemográficas y de salud relacionadas con el estrés laboral en un contexto sanitario: edad, sexo, estado civil, número de hijos, nivel de estudios finalizado, meses de ejercicio profesional (durante su vida laboral), meses de labor en el lugar de trabajo actual, meses de trabajo durante la pandemia producida por el virus Sars-CoV-2 (desde marzo hasta octubre de 2020), número de personas a su cargo (dentro del contexto laboral), otras actividades profesionales (independientes de su labor en la unidad de salud donde trabaja), horas semanales de actividad física, enfermedades físicas actuales (si padece o no, y cuáles), enfermedades mentales actuales (si padece o no, y cuáles) y patologías relacionadas con la enfermedad COVID-19 (problemas de salud cuya aparición está relacionada con la situación de pandemia, según el profesional).

\section{Cuestionario de Estrés Laboral (JSS) (7).}

Esta herramienta mide, en adultos, en el lapso de 15 a 20 minutos, tanto el nivel general de estrés laboral como la severidad y la frecuencia con que ocurren 30 fuentes genéricas de estrés, dando lugar a tres escalas y seis subescalas. A continuación, se transcribe la explicación que el autor aporta sobre cada medida y su finalidad, tal como figura en el manual del instrumento (pp.
20-21).

\section{Escalas:}

JS-X. Índice de Estrés laboral (Job Stress Index). El índice de Estrés laboral (JS-X) proporciona una estimación del nivel global de estrés laboral que experimenta un individuo en su contexto de trabajo. Combina las puntuaciones de severidad y frecuencia de todos los ítems del JSS.

JS-S. Severidad del Estrés laboral (Job Stress Severity). La escala de Severidad del Estrés laboral (JS-S) indica la puntuación media de severidad percibida por el individuo en las 30 situaciones estresantes del JSS. Tales puntuaciones están basadas en la comparación de cada uno de los 29 ítems de severidad ( 2 a 30) con el estresor estándar (ítem 1) al que le ha sido asignada una puntuación intermedia de 5 .

JS-F. Frecuencia del Estrés laboral (Job Stress Frecuency). La escala de Frecuencia del Estrés laboral (JS-F) indica la frecuencia media con la que las 30 situaciones estresantes del JSS se han dado en los últimos 6 meses (ítems 31 a 60).

Subescalas de Presión laboral y Falta de apoyo de la organización:

JP-X. Índice de Presión laboral (Job Pressure Index). El índice de Presión laboral (JP-X) evalúa (combinando la severidad y la frecuencia) el estrés laboral experimentado por un individuo atribuible a la presión procedente de su trabajo, tal como puede ser el trabajar tiempo extra, el cumplir plazos de finalización o el papeleo excesivo. Estos 10 estresores reflejan aspectos estresantes de la estructura, el diseño o las obligaciones del trabajo.

JP-S. Severidad de la Presión laboral (Job Pressure Severity). Esta subescala evalúa el nivel medio de severidad percibida respecto a los 10 estresores del JSS más directamente relacionados con la presión en el trabajo.

JP-F. Frecuencia de la Presión laboral (Job Pressure Frecuency). Esta subescala evalúa la frecuencia media de los 10 estresores del JSS más 
directamente relacionados con la presión en el trabajo.

LS-X. Índice de Falta de apoyo de la organización (Lack of Organizational Support Index). El índice de Falta de apoyo de la organización (LS-X) evalúa (combinando la severidad y la frecuencia) la cantidad de estrés laboral atribuible a la falta de apoyo de la organización, tal y como puede ser la dificultad para acceder a los superiores, a los compañeros de trabajo poco motivados y a la falta de oportunidades de promoción. Estos 10 estresores reflejan acontecimientos que implican a otras personas (p. ej., dificultades con los superiores y compañeros) o políticas y procedimientos organizacionales, más que aspectos del trabajo en sí mismos.

LS-S. Severidad de la Falta de apoyo de la organización (Lack of Organizational Support Severity). Esta subescala evalúa el nivel medio de severidad percibida respecto a los 10 estresores del JSS más directamente relacionados con la Falta de apoyo de la organización.

LS-F. Frecuencia de la Falta de apoyo de la organización (Lack of Organizational Support Frecuency). Esta subescala evalúa la frecuencia media con la que se producen los 10 estresores del JSS más directamente relacionados con la Falta de apoyo de la organización.

Los coeficientes de fiabilidad superaron el 0,85 en los nueve indicadores, mientras que la estructura factorial mostró un ajuste moderado con respecto a la de la versión original, que reveló dos factores: Presión laboral y Falta de apoyo de la organización. Para el procesamiento de las respuestas, se utilizaron los baremos españoles correspondientes al Grupo III (7) (que abarca las profesiones de mayor carga intelectual y responsabilidad dentro de una organización) de los apéndices del cuestionario de estrés laboral, los cuales indican los valores de percentiles $(\mathrm{Pc})$ en los que se encuentran los resultados de cada escala y desde donde se puede apreciar las diferencias entre los valores a analizar (p. 80).

\section{Procedimiento}

Una vez comprobados los criterios de inclusión, se explicaron las características del estudio a cada participante, quien firmó el consentimiento informado antes de iniciar la aplicación del protocolo de evaluación. La participación de cada profesional fue a título personal, no institucional. El trabajo de campo fue realizado entre septiembre y octubre de 2020, ya que el instrumento de estrés laboral JSS tiene la capacidad de evaluar a partir de los 6 meses los niveles de estrés, en total, cada evaluación individual tomó entre 45 y 60 minutos. No se presentaron inconvenientes durante la aplicación del protocolo de evaluación a la muestra de estudio. Las respuestas de cada cuestionario fueron registradas, procesadas y consolidadas en una base de datos.

\section{Análisis}

Se aplicaron estadísticos descriptivos para presentar las características sociodemográficas, de salud y de estrés laboral, para la muestra total. Además, se realizaron comparaciones de medias de muestras independientes ( $t$ de student) entre los resultados obtenidos por el personal sanitario y por grupos de edades en cada uno de los nueve indicadores del Cuestionario de estrés laboral, estableciendo niveles de significancia para valores $\mathrm{p}<0,05$. Finalmente, los datos fueron procesados utilizando el paquete estadístico SPSS.22 para Windows (20).

\section{RESULTADOS}

Los datos sociodemográficos de la muestra (ver Tabla 1) revelan que en las variables que mide el cuestionario sociodemográfico se encontró que dentro de los encuestados la mayor cantidad fueron mujeres (54\%), así mismo los encuestados están entre edades comprendidas desde los 20 a los 65 años (M (media) = 30,02 años; DT (desviación típica) $=10,520$ años), dividida en dos grupos: 22 médicos/as ( $\mathrm{M}=34,14$ años; $\mathrm{DT}=11,247 \mathrm{años}) \mathrm{y}$ 19 enfermeros/as $(M=25,26$ años; $\mathrm{DT}=07,332$ años). También se observaron resultados de salud relacionados con el estrés laboral, en donde los médicos/as puntúan más alto que los enfermeros/as.

Respecto a las variables del Cuestionario de Estrés Laboral, que miden el nivel general de estrés 
laboral, mediante tres escalas y seis subescalas (ver Tabla 2), los análisis revelan diferencias significativas entre las puntuaciones obtenidas por médicos/as y enfermeros/as (ver equivalencia de puntuaciones directas en percentiles (Pc), entre paréntesis, para apreciar la magnitud del impacto del conjunto de estresores). Para el Índice de Estrés laboral (Pc 85 (35,00-38,89) vs Pc 65 $(24,87-27,23))$ y su subescala severidad del Estrés laboral (Pc $75(6,23-6,40)$ vs Pc $50(5,43-5,59))$; para el Índice de Presión laboral (Pc 90 (44,30$50,79$ vs Pc $70(29,70-32,39))$ y su subescala severidad de la presión laboral (Pc 75 (6,30-6,49 Vs Pc 50 (5,40-5,59)); y para el Índice de Falta de apoyo de la organización (Pc $80(34,42-38,59)$ Vs Pc 60 $(22,10-24,79))$.

En la tabla 3 se pudo apreciar diferencias significativas entre las puntuaciones obtenidas por grupos de edad entre las personas menores o iguales a 30 años y para los mayores a 31 años para el Índice de Estrés laboral con los mismos percentiles Pc 85 y Pc 65, Índice de frecuencia de estrés laboral (Pc70 $(4,53-4,82)$ vs Pc85 $(5,53-5,99))$, Índice de presión laboral (Pc $75(32,40-35,49)$ vs Pc 90 (44,30-50,79)) y para el Índice de falta de apoyo a la organización (Pc $65(24,80-27,89)$ vs Pc $80(34,42-38,59))$.

\begin{tabular}{|c|c|c|c|}
\hline \multirow[t]{2}{*}{ Variables } & $\begin{array}{l}\text { Muestra total } \\
\qquad \mathrm{N}=41\end{array}$ & $\begin{array}{l}\text { Médicos/as } \\
n=22\end{array}$ & $\begin{array}{c}\text { Enfermeros/as } \\
n=19\end{array}$ \\
\hline & f o M (\% o DT $)$ & f o $M(\%$ o DT $)$ & f о $\mathrm{M}(\%$ o DT $)$ \\
\hline Edad & $30,02(10,520)$ & $34,14(11,247)$ & $25,26(7,332)$ \\
\hline \multicolumn{4}{|l|}{ Sexo } \\
\hline Hombres & $19(46)$ & $10(45)$ & $9(47)$ \\
\hline Mujeres & $22(54)$ & $12(55)$ & $10(53)$ \\
\hline \multicolumn{4}{|l|}{ Estado civil } \\
\hline Soltero & $29(71)$ & $13(59)$ & $16(84)$ \\
\hline Unión libre & $2(05)$ & $2(09)$ & \\
\hline Casado & $10(24)$ & $7(32)$ & $3(16)$ \\
\hline Número de hijos & $0,59(0,921)$ & $0,73(1,077)$ & $0,42(0,692)$ \\
\hline \multicolumn{4}{|l|}{ Nivel de estudios finalizado } \\
\hline Tercer nivel & $34(83)$ & $17(77)$ & $17(89)$ \\
\hline Cuarto nivel & $7(17)$ & $5(23)$ & $2(11)$ \\
\hline Meses de ejercicio profesional & $56,39(100,377)$ & $83,45(121,929)$ & $25,05(55,802)$ \\
\hline Meses de labor en el lugar de trabajo actual & $20,15(25,212)$ & $27,50(27,885)$ & $11,63(19,044)$ \\
\hline Meses de trabajo durante la COVID-19 & $5,34(1,559)$ & $5,27(1,549)$ & $5,42(1,610)$ \\
\hline Número de personas a su cargo & $5,76(22,597)$ & $10,27(30,407)$ & $0,53(1,504)$ \\
\hline \multicolumn{4}{|l|}{ Otras actividades profesionales } \\
\hline No & $31(76)$ & $15(68)$ & $16(84)$ \\
\hline Sí & $10(24)$ & $7(32)$ & $3(16)$ \\
\hline Horas semanales de actividad física & $2,86(3,338)$ & $2,18(2,575)$ & $3,65(3,976)$ \\
\hline \multicolumn{4}{|l|}{ Enfermedades físicas actuales } \\
\hline No & $30(73)$ & $13(59)$ & $17(89)$ \\
\hline Sí & $11(27)$ & $9(41)$ & $2(11)$ \\
\hline \multicolumn{4}{|l|}{ Enfermedades mentales actuales } \\
\hline No & $32(78)$ & $15(68)$ & $17(89)$ \\
\hline Sí & $9(22)$ & $7(32)$ & $2(11)$ \\
\hline \multicolumn{4}{|l|}{ Patologías relacionadas con la COVID-19 } \\
\hline No & $5(12)$ & $2(09)$ & $3(16)$ \\
\hline Sí & $36(88)$ & $20(91)$ & $16(84)$ \\
\hline
\end{tabular}

Tabla 1. Variables sociodemográficas y de salud

Nota. F (Frecuencia), M (Media), \% (Porcentaje), DT (Desviación Típica) Elaboración: los autores 


\begin{tabular}{|c|c|c|c|c|c|c|}
\hline \multirow{2}{*}{$\begin{array}{c}\text { Escalas/ } \\
\text { Subescalas }\end{array}$} & $\begin{array}{c}\text { Muestra total } \\
\mathrm{N}=\mathbf{4 1}\end{array}$ & $\begin{array}{c}\text { Médicos/as } \\
\mathrm{n}=\mathbf{2 2}\end{array}$ & $\begin{array}{c}\text { Enfermeros/as } \\
\mathrm{n}=\mathbf{1 9}\end{array}$ & \multirow{2}{*}{ Valor p } & \multirow{2}{*}{$\begin{array}{c}\text { Inferior } \\
\mathbf{9 5 \%} \text { CI }\end{array}$} & \multirow{2}{*}{ Superior 95\% CI } \\
\cline { 2 - 4 } & $\mathrm{M}(\mathrm{DT})$ & $\mathrm{M}(\mathrm{DT})$ & $\mathrm{M}(\mathrm{DT})$ & & & \\
\hline JS-X & $32,709(15,450)$ & $38,002(15,112)$ & $26,580(13,789)$ & $\mathbf{0 , 0 1 6}^{*}$ & 2,226 & 20,618 \\
\hline JS-S & $6,009(1,351)$ & $6,399(1,169)$ & $5,558(1,436)$ & $\mathbf{0 , 0 4 5}^{*}$ & 0,018 & 1,664 \\
\hline JS-F & $4,968(1,770)$ & $5,429(1,760)$ & $4,435(1,671)$ & 0,073 & $-0,096$ & 2,082 \\
\hline JP-X & $39,628(19,529)$ & $45,875(19,178)$ & $32,395(17,762)$ & $\mathbf{0 , 0 2 6}^{*}$ & 1,737 & 25,224 \\
\hline JP-S & $6,376(1,588)$ & $6,832(1,457)$ & $5,847(1,606)$ & $\mathbf{0 , 0 4 6}^{*}$ & 0,017 & 1,952 \\
\hline JP-F & $5,790(2,069)$ & $6,305(1,929)$ & $5,195(2,115)$ & 0,087 & $-0,168$ & 2,388 \\
\hline LS-X & $29,847(14,523)$ & $35,101(14,459)$ & $23,763(12,328)$ & $\mathbf{0 , 0 1 1 ^ { * }}$ & 2,775 & 19,901 \\
\hline LS-S & $5,824(1,347)$ & $6,190(1,188)$ & $5,400(1,426)$ & 0,060 & $-0,036$ & 1,615 \\
\hline LS-F & $4,615(1,798)$ & $5,036(1,759)$ & $4,126(1,762)$ & 0,107 & $-0,205$ & 2,025 \\
\hline
\end{tabular}

Tabla 2. Medias de las puntuaciones directas para las escalas y subescalas del Cuestionario de Estrés Laboral con respecto a la profesión.

Nota. JS-X (Îndice de Estrés laboral), JS-S (Severidad del Estrés laboral), JS-F (Frecuencia del Estrés laboral), JP-X (Índice de Presión laboral), JP-S (Severidad de la Presión laboral), JP-F (Frecuencia de la Presión laboral), LS-X (Índice de Falta de apoyo de la organización), LS-S (Severidad de la Falta de apoyo de la organización), LS-F (Frecuencia de la Falta de apoyo de la organización). ${ }^{*} \mathrm{p}<0.05$ : presentan diferencias estadísticamente significativas

Elaboración: los autores.

\begin{tabular}{|c|c|c|c|c|c|c|}
\hline Escalas/ & Muestra total & $\begin{array}{c}\text { Menores o } \\
\text { igual a } 30 \\
\text { años }\end{array}$ & Mayores a 30 años & \multirow{4}{*}{ Valor $\mathrm{p}$} & \multirow{4}{*}{$\begin{array}{l}\text { Inferior } \\
95 \% \text { CI }\end{array}$} & \multirow{4}{*}{$\begin{array}{c}\text { Superior } 95 \% \\
\text { CI }\end{array}$} \\
\hline \multirow[t]{3}{*}{ Subescalas } & $\mathrm{N}=\mathbf{4 1}$ & $\mathrm{n}=\mathbf{2 8}$ & $\mathrm{n}=13$ & & & \\
\hline & M & M & M & & & \\
\hline & (DT) & (DT) & (DT) & & & \\
\hline JS-X & $\begin{array}{c}32,709 \\
(15,450)\end{array}$ & $\begin{array}{c}28,781 \\
(12,743)\end{array}$ & $\begin{array}{l}41,1685 \\
(17,798)\end{array}$ & $0,015^{*}$ & $-22,222$ & $-2,551$ \\
\hline \multirow{2}{*}{ JS-S } & \multirow{2}{*}{$6,009(1,351)$} & 5,793 & 6,473 & \multirow{2}{*}{0,136} & \multirow{2}{*}{$-1,581$} & \multirow{2}{*}{0,222} \\
\hline & & $(1,406)$ & $(1,133)$ & & & \\
\hline \multirow{2}{*}{ JS-F } & \multirow{2}{*}{$4,968(1,770)$} & 4,600 & 5,761 & \multirow{2}{*}{$0,049^{\star}$} & \multirow{2}{*}{$-2,318$} & \multirow{2}{*}{$-0,004$} \\
\hline & & $(1,379)$ & $(2,272)$ & & & \\
\hline JP-X & $\begin{array}{c}39,628 \\
(19,529)\end{array}$ & $\begin{array}{c}34,877 \\
(17,015)\end{array}$ & $\begin{array}{c}49,861 \\
(21,299)\end{array}$ & $0,02^{\star}$ & $-27,502$ & $-2,466$ \\
\hline \multirow{2}{*}{ JP-S } & \multirow{2}{*}{$6,376(1,588)$} & 6,096 & 6,976 & \multirow{2}{*}{0,099} & \multirow{2}{*}{$-1,934$} & \multirow{2}{*}{0,173} \\
\hline & & $(1,541)$ & $(1,577)$ & & & \\
\hline \multirow{2}{*}{ JP-F } & \multirow{2}{*}{$5,790(2,069)$} & 5,396 & 6,638 & \multirow{2}{*}{0,073} & \multirow{2}{*}{$-2,606$} & \multirow{2}{*}{0,122} \\
\hline & & $(1,729)$ & $(2,529)$ & & & \\
\hline LS-X & $\begin{array}{c}29,847 \\
(14,523)\end{array}$ & $\begin{array}{c}26,567 \\
(12,500)\end{array}$ & $\begin{array}{c}36,909 \\
(16,501)\end{array}$ & $0,032^{\star}$ & $-19,746$ & $-0,935$ \\
\hline \multirow{2}{*}{ LS-S } & \multirow{2}{*}{$5,824(1.347)$} & 5,692 & 6,1054 & \multirow{2}{*}{0,368} & \multirow{2}{*}{$-1,329$} & \multirow{2}{*}{0,504} \\
\hline & & $(1,461)$ & $(1,057)$ & & & \\
\hline \multirow{2}{*}{ LS-F } & \multirow{2}{*}{$4,615(1,798)$} & 4,278 & 5,338 & \multirow{2}{*}{0,079} & \multirow{2}{*}{$-2,247$} & \multirow{2}{*}{0,127} \\
\hline & & $(1,497)$ & $(2,213)$ & & & \\
\hline
\end{tabular}

Tabla 3. Medias de las puntuaciones directas para las escalas y subescalas del Cuestionario de Estrés Laboral con respecto a grupos de edad

Nota. JS-X (Îndice de Estrés laboral), JS-S (Severidad del Estrés laboral), JS-F (Frecuencia del Estrés laboral), JP-X (Índice de Presión laboral), JP-S (Severidad de la Presión laboral), JP-F (Frecuencia de la Presión laboral), LS-X (Índice de Falta de apoyo de la organización), LS-S (Severidad de la Falta de apoyo de la organización), LS-F (Frecuencia de la Falta de apoyo de la organización). ${ }^{*} \mathrm{p}<0.05$ : presentan diferencias estadísticamente significativas; se tomo 30 años por ser la media de la edad en el grupo de estudio.

Elaboración: los autores. 


\section{DISCUSIÓN}

Los resultados obtenidos confirman las hipótesis planteadas, pues ambos tipos de profesionales revelan niveles de estrés laboral según la escala de la metodología aplicada, haciendo referencia a la tabla de baremos del apéndice del JSS (7) (Pc 5059 bajo, Pc 60-74 moderado, Pc75 o superior alto), los/as médicos/as presentan puntuaciones más altas que los/as enfermeros/as en los nueve parámetros que mide el cuestionario de estrés laboral (7). Si bien en términos estadísticos los hallazgos indican lo referido, desde la perspectiva clínica y laboral es indispensable considerar la magnitud del efecto de los estresores (trabajar horas extras, falta de oportunidades de promoción, equipamiento insuficiente o de pobre calidad entre otros) en ambos tipos de profesionales, por sus implicaciones en la salud laboral y el desempeño profesional, además, la estructura psicométrica del cuestionario de estrés laboral (JSS) sugiere poner énfasis en los resultados que arrojan el Índice de Estrés laboral, por ser una medida global del estrés laboral que experimenta un individuo (se trata de la combinación de la severidad más la frecuencia del estrés laboral que experimenta un individuo en su contexto de trabajo); el Índice de Presión laboral, por ser una medida específica del estrés atribuible a la presión del entorno laboral (se trata de la combinación de la severidad más la frecuencia de la presión laboral experimentada por un individuo atribuible a la presión procedente de su trabajo); y el Índice de falta de apoyo de la organización, por una medida del nivel de estrés laboral atribuible a la falta de apoyo de la organización (se trata de la combinación de la severidad más la frecuencia de la cantidad de estrés laboral atribuible a la falta de apoyo de la organización). Por esta razón, la discusión se centra en los hallazgos más relevantes en estos tres índices, aportando, entre paréntesis, la equivalencia de la media de las puntuaciones directas obtenidas en cada índice o subescala, para médicos/as y enfermeros/as, en percentiles, de acuerdo con su grupo normativo de comparación, y su relación con algunas variables sociodemográficas y de salud.

En primer lugar, en el índice de estrés laboral (Pc 85 vs Pc 65) se evidencia que los/as médicos/as puntúan más alto que los/as enfermeros/as. Exis- ten diversas explicaciones a estas diferencias, como son tener mayor experiencia profesional, llevar más tiempo en el lugar de trabajo actual y contar con más personal bajo su dirección. A esto se suman las funciones y las responsabilidades asociadas a su cargo, que implican toma de decisiones, respuesta inmediata y gestión adecuada de los recursos en una situación compleja como la que ha generado la pandemia por la enfermedad COVID-19. También hay que considerar la edad media de este grupo y la carga añadida de responsabilidad de tener una familia y realizar menos actividad deportiva física, factores que pueden justificar que presenten más patologías físicas (especialmente, hipertensión y problemas respiratorios) y mentales (especialmente, ansiedad, depresión y trastornos del sueño) en el momento de la evaluación. En relación con un estudio realizado en una de las regiones italianas más afectadas, donde se mostraron que los trabajadores sanitarios de primera línea revelan niveles altos de síntomas depresivos debido a que se encuentran expuestos a situaciones de alto riesgo, que pueden hacer que se sienten inseguros en el trabajo, la implacable propagación del virus, la falta descanso suficiente y la amenaza permanente de infectarse (21).

En segundo lugar, el índice de presión laboral (Pc 90 vs Pc 70) es coherente con los resultados expuestos anteriormente, al demostrar que los médicos/as perciben niveles de presión más altos. Las condiciones de trabajo impuestas por el Ministerio de Salud del Ecuador ante la emergencia sanitaria ha requerido un esfuerzo descomunal por parte del personal sanitario y una disponibilidad total: jornadas intensivas de trabajo, horas extras de trabajo, falta de recursos para atender satisfactoriamente la demanda laboral asociada a su función, escaso control de la carga laboral, imposibilidad de programar y distribuir adecuadamente el trabajo, dirigir grupos de trabajo en situaciones complejas, etc. (22). Estas circunstancias afectan considerablemente a ambos tipos de profesionales, pero en intensidad distinta. Sin embargo, tanto médicos/as como enfermeros/as reportan diversas alteraciones de salud que, en su opinión, están asociadas directamente al periodo de trabajo en el marco de la pandemia por COVID-19 y al estilo de vida que ha impuesto a la 
sociedad ecuatoriana (especialmente, trastornos del sueño, cefaleas, ansiedad, depresión, irritabilidad y diversas manifestaciones psicosomáticas), desde marzo de 2020 hasta la fecha. En comparación con otro estudio donde los resultados presentaron una alta carga psicológica y prevalencia de síntomas de salud, una proporción considerable de participantes tenía síntomas de depresión ansiedad, insomnio y angustia. Enfermeras, trabajadores de primera línea, y aquellos en $\mathrm{Wu}-$ han informaron haber experimentado niveles de síntomas más graves de depresión, ansiedad, insomnio y angustia; depresión grave entre médicos y enfermeras; ansiedad severa entre hombres y mujeres; insomnio severo entre los trabajadores de primera línea, frente a los de la segunda línea (23).

Finalmente, el Índice de Falta de apoyo de la organización (Pc 80 vs Pc 60) indica que los médicos/as perciben menos apoyo por parte de la organización que los/as enfermeros/as. En la línea de lo discutido anteriormente, es posible que estos profesionales hayan experimentado, en esta fase de pandemia por la enfermedad COVID-19, dificultades para acceder a sus superiores y compañeros de trabajo o ver satisfechas sus demandas por parte del Ministerio de Salud Pública. Este dato puede estar ligado a la carga laboral que asumen, en gran medida por la brecha en materia de profesionales sanitarios necesarios para atender las necesidades de la población, en una situación de normalidad, realidad que se potencia en una emergencia sanitaria (12). Esta relación entre estrés laboral y patologías físicas y mentales ha sido referida en otros estudios, donde se evidencia que los niveles elevados de estrés, presión laboral y falta de apoyo se relacionan con el agotamiento físico y mental en el personal de salud (24-28).

Los hallazgos del presente estudio confirman la incipiente información que se tiene en esta materia durante la pandemia por la enfermedad COVID-19, particularmente en nuestro país, a la vez que fomentan una vía de investigación crucial para la gestión de talento humano del Sistema Nacional de Salud, que se ve obligado a tomar medidas necesarias para evitar el deterioro físico y mental innecesario del personal sanitario, res- ponsable de la atención directa al usuario. Esto requiere la revisión y atención inmediata de las necesidades en materia de infraestructura, de personal, de equipos y de insumos, junto con la aplicación de una estrategia inteligente de gestión del talento humano, que repercutirá favorablemente en la productividad a corto, mediano y largo plazos del personal y en los indicadores de eficiencia y eficacia de la organización y, particularmente, de satisfacción del usuario.

La principal fortaleza de este estudio radica en que es la primera vez, que se lleva a cabo un análisis del nivel de estrés en profesionales sanitarios del Sistema Nacional de Salud (médicos/as y enfermeros/as), que han brindado, desde que se inició la emergencia sanitaria por la enfermedad COVID-19, atención personalizada al usuario. Sin embargo, se presentan como limitaciones evidentes el tamaño de muestra y la falta de inclusión de otros profesionales de la salud, como psicólogos, obstetras, odontólogos/as y tecnólogos/as médicos/as, en cuanto al primer nivel de atención en salud.

\section{CONCLUSIONES}

Ante la emergencia sanitaria por la enfermedad COVID-19, el personal sanitario evaluado, médicos/as y enfermeros/as se encuentran expuestos a situaciones que repercuten de manera negativa en la salud física y mental. Los/as médicos/as presentan puntuaciones más elevadas de niveles de estrès y clínicamente significativas que los/as enfermeros/as en los nueve parámetros que mide el cuestionario de estrés laboral: índice de estrés laboral, severidad del estrés laboral, frecuencia del estrés laboral, índice de presión laboral, severidad de la presión laboral, frecuencia de la presión laboral, índice de falta de apoyo de la organización, severidad de la falta de apoyo de la organización y frecuencia de la falta de apoyo de la organización, así como también, en algunas escalas dependiendo de los grupos de edad de los participantes en el estudio, siendo los que tienen una edad mayor a 30 años los que presentan mayor estrés laboral según la escala utilizada Para dar un impulso significativo a esta línea de investigación, futuros estudios deberán, subsanar estas limitaciones e incluir un conjunto más am- 
plio de variables que pueden estar relacionadas, directa e indirectamente, con el estrés laboral del profesional sanitario, dentro y fuera de su entorno laboral.

$\mathrm{R}$ eferencias

1. Rodríguez D. Consecuencias del síndrome de burnout en el trabajo y estrategias de prevención de riesgos para la seguridad y salud laboral. Noticias. 2020; 3(5).

2. Chambel M, Peiró J. Do values related to job control matter in predicting well-being at work? Revista de psicología de la salud. 2005; 17: p. 67-81.

3. Eller N, Netterstrøm B, Gyntelberg F, Kristensen T, Nielsen F, Steptoe A, et al. Work-related psychosocial factors and the development of ischemic heart disease: a systematic review. Cardiology in review. 2009; 17: p. 83-97.

4. Gil-Monte P. Situación actual y perspectiva de futuro en el estudio del estrés laboral: la Psicología de la salud ocupacional. Unidad de Investigación Psicosocial de la Conducta Organizacional (UNIPSICO). Información Psicológica. 2010;: p. 63-86.

5. Karasek R, Brisson C, Kawakami N, Houtman I, Bongers P, Amick B. The Job Content Questionnaire (JCQ): an instrument for internationally comparative assessments of psychosocial job characteristics. comparative assessments of psychosocial job characteristics. Journal of occupational health psychology. 1998; 3(322).

6. Hurrel J, McLaney A. Exposure to job stress: A new psychometric instrument. Scandinavian Journal of Work, environment \& Health. 1988; 14: p. 27-28.

7. Catalina C. Cuestionario de estrés laboral. Adaptación española del Job Stress Survey (Spielberger y Vagg, 1991). Madrid: Tea Ediciones. 2010.

8. Spielberger C, Vagg P. Job Stress Survey (JSS). Professional manual. Odesa, Florida:; 1991.

9. Pérez J. ¿Qué es el estrés laboral y como medirlo? Salud Uninorte. 2019; 35: p. 156-185.

10. Collin-Orellana A, Vásquez-Cassinelli J, Duque-Córdova L, Espinoza-Samaniego C, AriasUlloa C, Escobar-Segovia K. Improvement in service quality by analyzing the relationship between psychosocial risks and labor engagement in a vertical transport equipment maintenance company. In 18th LACCEI International Multi-Conference for Engineering, Education Caribbean Conference for Engineering and Technology: "'Engineering, Integration, and Alliances for a Sustainable Development"' LACCEI; 2020; Buenos Aires.

11. Mamani-Encalada A, Obando-Zegarra R, Uribe-Malca A, Vivanco-Tello M. Factores que desencadenan el estrés y sus consecuencias en el desempeño laboral en emergencia, 3. Revista Peruana de Obstetricia y Enfermería. 2007; 3.

12. Fernandes M, Alencar-Ribeiro A. Salud mental y estrés ocupacional en trabajadores de la salud a la primera línea de la pandemia de COVID-19. Revista Cuidarte. 2020;: p. 12-22.

13. Dongil-Collado E. Pautas para detectar el estrés laboral. Gestión Práctica de Riesgos Laborales. Universidad Católica de Valencia. 2009; 56(48).

14. Patlan J. ¿Qué es el estrés laboral y cómo medirlo? Revista Salud Uninorte. 2019;: p. 156-184.

15. Castillo S. El Síndrome de "Burn Out" o Síndrome de Agotamiento Profesional. Med. leg. Costa Rica. 2001; 17(2): p. 11-14.

16. Castillo G, Rosas L, Cajías L, Escobar-Segovia K. Identidficcación del Sindrome de Burnout en personal médico del área de emergencias en un hospital de segundo nivel en Machala-Ecuador. Revista Ciencia y Salud Virtual. 2019; 2: p. 79-89.

17. Badahdah A, Khamis F, Al-Mahyijari N, Al-Balushi M, Al-Hatmi H, Al-Salmi I, et al. The mental health of health care workers in Oman during the COVID-19 pandemic. The International Journal of Social Psychiatry. 2020.

18. Ybaseta-Medina J, Becerra-Canales B. El personal de salud en la pandemia por COVID-19. Revista Médica Panacea. 2020;: p. 72-73.

19. Salopek-Žiha D, Hlavati M, Gvozdanović Z, Gašić M, Placento H, Jakić H, et al. Differences in 
distress and coping with the COVID-19 stressor in nurses and physicians. Psychiatria Danubina. 2020; 32(2): p. 287-293.

20. IBMCorp. IBM SPSS Statistics for Windows (version 22.0). 2013..

21. Di Tella M, Romeo A, Benfante A, Castelli L. Salud mental de los trabajadores de la salud durante la pandemia de COVID-19 en Italia. Revista de evaluación en la práctica clínica. 2020; 26(6): p. 1583-1587.

22. Xu H, Johnston A, Greenslade J, Wallis M, Elder E, Abraham L, et al. Stressors and coping strategies of emergency department nurses and doctors: A cross-sectional study. Australasian emergency care. 2019;: p. 180-186.

23. Lai J, Ma S, Wang Y, Cai Z, Hu J, Wei N, et al. Factors associated with mental health outcomes among health care workers exposed to coronavirus disease 2019. JAMA network open. 2020; 3(3).

24. Basu S, Qayyum H, Mason S. Occupational stress in the ED: a systematic literature review. Emergency medicine journal: EMJ. 2017;: p. 44-47.

25. Espinoza-Guerra E, Quimí-Espinosa J, Escobar-Segovia K, Camacho-Polo I. Riesgos psicosociales y satisfacción laboral en empresas que prestan servicios de salud ocupacional: un estudio en la ciudad de Guayaquil. Revista Psicología UNEMI. 2020; 7: p. 21-39.

26. Gómez-Salgado J, Andrés-Villas M, Domínguez-Salas S, Díaz-Milanés S, Ruiz-Frutos C. Related Health Factors of Psychological Distress During the COVID-19 Pandemic in Spain. International Journal of Environmental Research and Public Health. 2020; 17(3947).

27. García-Iglesias J, Gómez-Salgado J, Martín-Pereira J, Fagundo-Rivera J, Ayuso-Murillo D, Martínez-Riera J, et al. Impacto del SARS-CoV-2 (Covid-19) en la salud mental de los profesionales sanitarios: una revisión sistemática. Revista espanola de salud publica. 2020 julio; 94.

28. Gómez-Salgado J, Domínguez-Salas S, Romero-Martín M, Ortega-Moreno M, García-Iglesias J, Ruiz-Frutos C. Sense of coherence and psychological distress among healthcare workers during the COVID-19 pandemic in Spain. Sustainability (Switzerland). 2020 September; 12(17). 